\title{
CONFERENCE REPORT \\ Heart of the tropics: delivering evidence-based care for acute coronary syndromes in northern Australia
}

\author{
G Starmer ${ }^{1}$, R Schrale ${ }^{2}$ \\ ${ }^{1}$ Cairns Hospital, Cairns, Queensland, Australia \\ ${ }^{2}$ Townsville Hospital, Douglas, Queensland, Australia
}

Submitted: 6 March 2016; Revised: 11 July 2016; Accepted: 29 August 2016; Published: 6 November 2016

Starmer G, Schrale R

Heart of the tropics: delivering evidence-based care for acute coronary syndromes in northern Australia Rural and Remote Health 16: 3938. (Online) 2016

\author{
Available: http://www.rrh.org.au
}

\section{A B S T R A C T}

Acute coronary syndromes (ACS) are life-threatening medical emergencies that require urgent treatment, posing particular challenges for systems of health care in regional and remote parts of the world characterised by large distances and widely dispersed populations and healthcare facilities. Northern Australia is such an environment. The prevalence of cardiovascular risk factors, coronary artery disease and ACS (myocardial infarction and unstable angina) in northern Australia is amongst the highest in Australia. Despite the high burden of disease, appropriate healthcare services to address these important health challenges have been inadequate.

The Australian Commission on Safety and Quality in Health Care has released a Clinical Care Standard for Acute Coronary Syndromes and the National Heart Foundation of Australia has developed an ACS Capabilities Framework, which together define minimum standards of care regardless of the patient's location. Strategies such as uniform state-wide ACS clinical pathways provide guidance on how evidence-based care can be provided in a range of geographical settings and to all populations, including Indigenous Australians. The continuing evolution of cardiac catheter laboratories in Townsville, Cairns, Mackay and Darwin has facilitated improved treatment for ACS in northern Australia, and has supported the development of region-wide, integrated, multidisciplinary pathways of care.

Systems of care in ACS require consideration of the perspectives of the patient (from symptom onset to long-term secondary prevention of further events), the health system ('dissolving' traditional regional silos of care to enable a higher critical mass, greater cooperation, better communication and improved efficiency) and healthcare disciplines and services (including ambulance, retrieval, local health centres and local hospitals, tertiary centres, cardiac rehabilitation and general practice).

Key words: acute coronary syndrome, northern Australia, regional systems, system of care. 


\section{Context}

\section{Introduction}

Acute coronary syndromes (ACS: myocardial infarction and unstable angina) are life-threatening medical emergencies that require urgent treatment to save lives and prevent permanent damage to the heart in survivors. An integrated network of care to ensure guideline-based treatment and to minimise delay in providing definitive therapy is desirable $^{1}$, but considerable barriers exist to implementing such systems in regional and remote locations. Although the delivery of ACS care varies widely by country, the obstacles encountered by non-metropolitan regions are remarkably similar across health services globally.

Australia is the sixth-largest country in the world. The term 'northern Australia' has been used in policy-making and planning to refer to the land mass, amounting to about onethird of the country's total area, lying to the north of the Tropic of Capricorn ${ }^{2}$. Northern Australia is characterised by vast distances with a significantly dispersed population. Four major regional cities, Darwin, Cairns, Townsville and Mackay, provide tertiary-level cardiac services. Outside these centres, medical care is provided largely by rural generalists and small hospitals with limited facilities.

The authors convened two conferences, in Townsville in March 2014 and Cairns in August 2015, to explore how effective, integrated, multidisciplinary care can be provided to patients with ACS throughout northern Australia, regardless of their location. Each conference, held over two half-days and with about 20 participants, consisted of structured presentations followed by discussion. The participants included representatives of ambulance services, medical retrieval services, general practice, rural generalists in local hospitals, tertiary cardiac services, cardiac rehabilitation and data registry services.
This report is the authors' synthesis of the conferences. It summarises the challenges in implementing new national standards of ACS care in northern Australia and suggests pathways to improved care.

\section{Acute coronary syndromes: definition and significance}

ACS occur when blood flow through the coronary arteries to the myocardium is interrupted, usually as a result of atherosclerotic coronary artery disease. Based on ECG findings the events are initially classified as ST-elevation myocardial infarction (STEMI), non-ST-elevation myocardial infarction (NSTEMI) or unstable angina. NSTEMI and unstable angina are together referred to as non-ST-elevation ACS (NSTEACS). Early angiography is desirable to define the anatomy of the coronary artery lesions responsible for the event and to guide optimal treatment.

STEMI results from a complete occlusion of a coronary artery by a thrombus and can lead rapidly to death of myocardium in the area supplied by the vessel, and also to life-threatening cardiac arrhythmias. NSTEMI arises from partial occlusion of a coronary artery which is sufficient to cause injury to the myocardium. In unstable angina, partial occlusion of an artery leads to ECG changes and symptoms, without injury to the myocardium. About 70000 Australians are estimated to experience an ACS every year, and coronary artery disease accounts for about $15 \%$ of all deaths ${ }^{3}$

ACS requires an urgent response encompassing life support if needed, initial assessment and risk stratification, and definitive treatment of the occluded coronary artery to restore perfusion. Reperfusion in patients with STEMI can be delivered by fibrinolysis in the community or in hospital, or by primary percutaneous coronary intervention (PCI) delivered in a specialist cardiac catheter laboratory (eg balloon angioplasty followed by insertion of a stent). In a small number of cases patients will require urgent coronary artery bypass surgery. Management of patients with NSTEMI and unstable angina is guided by a careful initial risk assessment, and consideration of coronary angiography. In 
patients presenting with NSTEMI and high risk features on initial assessment, early coronary angiography $(<24 \mathrm{~h})$ is indicated. Well-established Australian and international clinical guidelines on the treatment of ACS are available ${ }^{1,4-6}$. The guidelines emphasise the necessity for timely reperfusion, and they increasingly recognise the benefits of treatment in a tertiary cardiac care centre with catheter laboratory facilities.

Follow-up care to reduce the risk of further events is an essential component in the continuum of care, as about half of all ACS occur in patients with a prior hospital admission for a myocardial infarction ${ }^{7}$.

\section{Issues}

\section{Discrepancy between ACS risks and ACS services}

People living in rural and remote Australia have more risk factors for cardiovascular disease, higher rates of cardiovascular disease-related hospital admission and are more likely to die of cardiovascular disease than those in metropolitan areas ${ }^{8}$. The further a person lives from a metropolitan centre, the higher the risk of hospitalisation for, and death from, cardiovascular disease. In 2011-12 the National Heart Foundation of Australia mapped 'hotspots' for heart disease across Australia, and found the prevalence of cardiovascular risk factors, coronary artery disease and ACS (myocardial infarction and unstable angina) in northern Australia to be amongst the highest in the country 9 . The prevalence of cardiovascular disease in the Cairns region, the most northern catchment in the state of Queensland, was $27.5 \%$.

The proportion of the population who are Indigenous Australians is high in northern Australia, and they carry a particular burden of cardiovascular disease. Compared with non-Indigenous Australians, Aboriginal and Torres Strait Islander peoples are $70 \%$ more likely to die from circulatory diseases ${ }^{10}$. Indigenous men are twice as likely, and Indigenous women three times as likely, to be hospitalised for ischaemic heart disease compared to non-Indigenous Australians, and they have an elevated prevalence of risk factors including hypertension, smoking and obesity.

Access to cardiology services in northern Australia has been limited, despite the high rates of cardiovascular disease in the region. For example, rates of hospital admission for PCI, whether elective or in response to an ACS, in north Queensland, the Northern Territory and the north of Western Australia, are amongst the lowest in Australia ${ }^{11}$.

\section{New standards of care define service requirements}

In 2014 the Australian Commission on Safety and Quality in Health Care (ACSQHC) released a Clinical Care Standard for Acute Coronary Syndromes ${ }^{12}$. Providing a benchmark against which services can be assessed, the standard aims to ensure that patients with ACS receive optimal treatment from the onset of symptoms through to discharge from hospital, including recognition of ACS, rapid assessment, early management and early initiation of a tailored rehabilitation plan. It states that, 'Central to the delivery of patient-centred care ... is an integrated, systems-based approach supported by health services and networks of services'. The standard applies to all Australians, without any suggestion that the quality of care should be compromised because of a patient's geographical location.

Six quality statements within the standard define a need for:

- immediate management guided by a documented chest pain assessment pathway

- $\quad$ early assessment including a 12-lead ECG with the results analysed by an experienced clinician within 10 minutes of the first emergency clinical contact

- timely reperfusion for patients with STEMI, in accordance with the time frames recommended by current guidelines (in general, PCI is recommended if the time from first medical contact to balloon inflation is anticipated to be less than 90 minutes, otherwise the patient is offered fibrinolysis) 
- $\quad$ risk stratification and management of patients with a NSTEACS based on a documented, evidence-based assessment of their risk of an adverse event

- consideration of coronary angiography, with a view to timely and appropriate coronary revascularisation, in patients with NSTEACS at intermediate or high risk of an adverse cardiac event

- development of an individualised care plan before patients with ACS leave hospital, addressing lifestyle modifications and medicines needed to manage their risk factors, psychosocial needs and referral to cardiac rehabilitation or another secondary prevention program.

In 2015 the National Heart Foundation of Australia released the Australian acute coronary syndromes capability framework, aiming 'to support the national delivery of evidence-based care for those experiencing acute coronary syndromes irrespective of where they live in Australia' (emphasis added) ${ }^{13}$. Designed to complement the ACSQHC standard and existing clinical guidelines, the framework recognises that ACS care is delivered in varying circumstances, ranging from pre-hospital emergency services to hospitals with an emergency service, hospitals with a dedicated emergency department, and tertiary cardiac centres. The framework defines which of the six ACSQHC standards can be delivered by each level of service; for example, pre-hospital emergency care can potentially deliver immediate management, early assessment and timely reperfusion.

\section{Challenges in implementing evidence-based care}

The ACSQHC Standards and Heart Foundation Framework establish the benchmark for ACS care in northern Australia. Geographical and logistical barriers exist that will make implementation of these standards difficult for rural and regional centres. Each major regional catchment has its own governance structure and is funded separately by health departments to provide services, hindering cooperation and discouraging a shared service model of care. However, evolving systems of ACS care in these regions have demonstrated the progress that can be achieved.
Significant steps include the development of cardiac catheter laboratories, staffed by experienced teams of clinicians and technicians, in Townsville, Cairns, Mackay and Darwin. The advent of these tertiary services has been one factor in driving the evolution of wider integrated, multidisciplinary systems of care that include ambulance services, retrieval services, general practitioners, community health centres and local hospitals.

In Queensland, integrated care has been further supported by the release of three clinical pathways on the management of possible cardiac chest pain, the management of a diagnosed ACS, and thrombolysis for STEMI ${ }^{14}$. The pathways provide clarity for all healthcare providers in managing patients' journeys from their first contact with medical services through to definitive treatment and discharge from hospital. Information included within the pathways includes details of medication; for example, in the first 24 hours after presentation with ACS, the pathway recommends prescription of aspirin, either clopidogrel or ticagrelor, a beta-blocker (in the absence of heart failure or heart block), and appropriate PRN medication such as glyceryl trinitrate, IV analgesia and an IV anti-emetic.

Initiatives supporting an integrated approach include the following:

- Queensland Ambulance Service paramedics can autonomously treat STEMI patients with fibrinolysis before transfer to hospital, or transport patients directly to a catheter laboratory for primary PCI if it can be achieved within the recommended time frame. The fibrinolysis protocol can also be initiated by GPs and nurses when appropriate.

- Catheter laboratories in northern Queensland encourage direct contact from GPs and community hospitals on the appropriate management of patients, including arrangements for timely transfer to the tertiary hospital when appropriate.

- Liaison with retrieval services assists in the timely transfer of patients with ACS to a tertiary cardiac facility, using available resources as effectively as 
possible to support evidence-based care for all patients.

It is logistically challenging to provide continuous catheter laboratory services for primary PCI in patients with ACS, and a critical mass of experienced staff is required before a sustainable service can reliably be offered. Large cardiac units in Australia's capital cities typically have at least six interventional cardiologists available to participate in on-call rosters, and access to a considerable resource of experienced nurses and technicians. In regional centres, on-call responsibilities are typically carried by two or three interventional cardiologists and a small number of nurses and technicians. Careful management is essential to ensure that a truly continuous service can be provided without undue and unsustainable demands on staff. The usual pattern has been for regional facilities to initially offer diagnostic or elective services during business hours, consolidating their experience and expertise before commencing time-critical services such as primary PCI. The four centres serving northern Australia, in Townsville, Cairns, Mackay and Darwin, are in varying stages of development, but each has had a marked impact on systems of care in the region it serves. Once a capacity has been developed, for example the provision of primary PCI for ACS patients on a continuous basis, the uptake of the service has been rapid.

Experience with cardiac care networks in South Australia also demonstrates what can be achieved in regional and remote locations. In a state also characterised by large distances and uneven population density, the Integrated Cardiovascular Clinical Network has been in place since 2001, aiming to reduce the discrepancy in cardiovascular outcomes for people in rural areas. Key elements include standardised risk stratification and evidencebased treatment protocols, point-of-care testing for troponin (a marker of myocardial necrosis) with central quality control, and access to a designated on-call consultant cardiologist to provide ECG interpretation and facilitation of transfer to metropolitan hospitals when necessary ${ }^{15}$. Patients requiring transfer from regional and rural areas are regarded as having the same 'rights' to a bed in a tertiary centre as a patient in that hospital's own emergency department. Implementation of the network has been associated with a $22 \%$ relative reduction in 30 -day mortality after ACS.

\section{Facilitating continuing care}

Follow-up care is an essential element in rehabilitation after ACS and in preventing recurrent cardiovascular events. Increasingly in northern Queensland referral to cardiac rehabilitation occurs automatically on an 'opt out' rather than 'opt in' basis. Close attention is paid to providing timely and relevant discharge summaries which will assist the patient's GP in providing essential long-term treatment to reduce the risk of recurrent cardiovascular events (secondary prevention). Such care, consistent with the secondary prevention guidelines of the National Heart Foundation of Australia $^{16}$, includes management of 'lifestyle' factors (such as diet, exercise, excess weight and smoking), and the use of a range of medications to reduce risks associated with hypertension, lipid abnormalities, thrombosis (including the use of dual antiplatelet therapy) and diabetes.

\section{Monitoring performance is essential}

Progress in the delivery of services is difficult to achieve unless current performance can be defined and reviewed. Quality standards such as the ACQSHC Standards and Heart Foundation ACS Framework provide benchmarks, but action is required to test how the system is performing.

In addition to local systems for quality assurance, initiatives such as the Queensland Cardiac Outcomes Registry (QCOR) offer a structure for resolving information barriers to the delivery of safe and effective care across an entire state. Developed in consultation with clinicians, for clinicians, the initial QCOR registries are accumulating and reporting on data on cardiac catheter laboratory procedures, cardiac surgery, heart failure, cardiac rehabilitation and echocardiography.

\section{Lessons learned}

\section{Pathway to improved ACS care in northern Australia}

Three themes emerged from the conferences regarding the features of systems of care that can deliver integrated, 
multidisciplinary, emergency responses to patients with ACS, regardless of their location:

- Patients require integrated, comprehensive and 'seamless' care from the time of their first contact with medical services through initial assessment and treatment, hospital admission, discharge, and longterm secondary prevention after the event.

- The health system must eliminate barriers that arise from bureaucratic regional divisions in areas characterised by low average population density and large distances. Such barriers can hinder collaboration, the development of a sustainable critical mass of facilities and staff, and effective communication between individuals and services.

- Healthcare disciplines and services, including ambulance, retrieval, local health centres and local hospitals, tertiary centres, cardiac rehabilitation and general practice, need an integrated and consistent approach when responding to patients with ACS.

From the conclusion of the first meeting, and strengthened further by the second meeting, an increase in clinical interaction, collaboration and communication between the service lines mentioned (first response, emergency, cardiologists) as well as between regional health services has been clearly evident. This has resulted in across-district implementation of a universal echocardiogram viewing and reporting installation for the three centres in northern Queensland, the development of a structured cardiac outreach program encompassing the entire northern Queensland region (yet to be implemented), and weekly teleconferencing between Cairns, Townsville and Mackay. Each health service has resolved to hold regular case conference and education meetings involving the Queensland Ambulance Service, and emergency departments.

\section{Conclusions}

ACS is a common, life-threatening medical emergency that often requires complex medical treatment. The logistics of delivering modern evidence-based care in regional, rural and remote Australia can be very challenging. Recent developments in systems of care in northern Australia demonstrate that integrated, comprehensive, multidisciplinary care can be provided, based on clear treatment pathways, engagement of healthcare providers at each step, and close communication between services. There has been a very considerable expansion in access to evidencebased care in recent years, but further progress is required to ensure that new standards of care can be implemented for all ACS patients, regardless of their location.

We recommend continuing efforts to 'dissolve' conventional geographical, bureaucratic and disciplinary boundaries, ensuring that such care can be provided. Experience with the management of ACS in these environments, now and in the future, can provide insights into how other heath challenges in northern Australia, and other regional and remote locations around the world, might be addressed. In addition, some of the principles will also be applicable to urgent, acute care in such locations, for example in the management of trauma, cerebrovascular accidents and obstetrics.

\section{Acknowledgments}

We gratefully acknowledge the invaluable contribution of all participants in the two conferences that formed the basis of this report. The logistics of the conferences were supported by AstraZeneca, which had no input into the selection of speakers or the content. Tony James, from CME Australia, provided medical writing support, funded by AstraZeneca.

\section{References}

1. Task Force on the management of ST-segment elevation acute myocardial infarction of the European Society of Cardiology (ESC), Steg PG, James SK, Atar D, Badano LP, Blömstrom-Lundqvist C, Borger MA, et al. ESC Guidelines for the management of acute myocardial infarction in patients presenting with ST-segment elevation. European Heart Journal 2012; 33: 2569-2619. 
2. Commonwealth of Australia. White paper on developing northern Australia. Frequently asked questions. (Internet) 2015. Available: http://industry.gov.au/ONA/WhitePaper/faqs.html (Accessed 16 June 2016).

3. Australian Institute of Health and Welfare. Australia's health 2014. Canberra: AIHW, 2014.

4. Acute Coronary Syndrome Guidelines Working Group. Guidelines for the management of acute coronary syndromes 2006. Medical Journal of Australia 2006; 184(8 Suppl.): S1-S32.

5. Roffi M, Patrono C, Collet JP, Mueller C, Valgimigli M, Andreotti F, et al. 2015 ESC Guidelines for the management of acute coronary syndromes in patients presenting without persistent ST-segment elevation: Task Force for the Management of Acute Coronary Syndromes in Patients Presenting without Persistent STSegment Elevation of the European Society of Cardiology (ESC). European Heart Journal 2015; 29 August. pii: ehv320. http://dx. doi.org/10.1016/j.rec.2015.10.009

6. Briffa TG, Hobbs MS, Tonkin A, Sanfilippo F, Hickling S, Ridout S, et al. Population trends of recurrent coronary heart disease event rates remain high. Circulation: Cardiovascular Quality and Outcomes 2011; 4: 107-113. http://dx.doi.org/10.1161/ circoutcomes.110.957944

7. National Rural Health Alliance and National Heart Foundation of Australia. Cardiovascular disease in rural Australia. Fact sheet. Melbourne: NRHA and NHFA, May 2015.

8. National Heart Foundation of Australia. Prevalence of cardiovascular disease (CVD) in Queensland. (Internet) 2014. Available: http://heart foundation.org.au/images/uploads/publications/2014HeatMaps_P revCVD_NAT.pdf (Accessed 16 June 2016).
9. Chew DP, Aroney CN, Aylward PE, Kelly AM, White H, Tideman P, et al. 2011 Addendum to the National Heart Foundation of Australia/Cardiac Society of Australia and New Zealand Guidelines for the management of acute coronary syndromes (ACS) 2006. Heart Lung and Circulation 2011; 20: 487 502. http://dx.doi.org/10.1016/j.hlc.2011.05.276

10. Australian Bureau of Statistics. Australian Aboriginal and Torres Strait Islander Health Survey: first results. ABS, 2013.

11. Australian Commission on Safety and Quality in Health Care and Australian Institute of Health and Welfare. Exploring healthcare variation in Australia: analyses resulting from an OECD study. Sydney: ACSQHC, 2014.

12. Australian Commission on Safety and Quality in Health Care. Acute coronary syndromes clinical care standard. Sydney: ACSQHC, 2014.

13. National Heart Foundation of Australia. Australian acute coronary syndromes capability framework. Melbourne: National Heart Foundation of Australia, 2015.

14. Queensland Health. ACS pathways 2015. (Internet) 2015. Available: https://www.health.qld.gov.au/caru/pathways/ pathways.asp (Accessed 16 June 2016).

15. Tideman PA, Tirimacco R, Senior DP, Setchell J, Huynh L, Tavella R, et al. Impact of a regionalised clinical cardiac support network on mortality among rural patients with myocardial infarction. Medical Journal of Australia 2014; 200: 157-160. http:// dx.doi.org/10.5694/mja13.10645

16. National Heart Foundation of Australia and the Cardiac Society of Australia and New Zealand. Reducing risk in heart disease: an expert guide to clinical practice for secondary prevention of coronary heart disease. Melbourne: National Heart Foundation of Australia, 2012. 